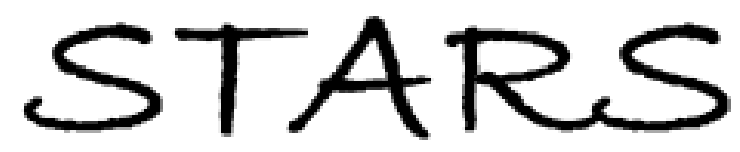

University of Central Florida

STARS

$1-1-2008$

\title{
Phonon-defect scattering in doped silicon by molecular dynamics simulation
}

M. Yao

T. Watanabe

P. K. Schelling

University of Central Florida

P. Keblinski

D. G. Cahill

See next page for additional authors

Find similar works at: https://stars.library.ucf.edu/facultybib2000

University of Central Florida Libraries http://library.ucf.edu

This Article is brought to you for free and open access by the Faculty Bibliography at STARS. It has been accepted for inclusion in Faculty Bibliography 2000 s by an authorized administrator of STARS. For more information, please contact STARS@ucf.edu.

\section{Recommended Citation}

Yao, M.; Watanabe, T.; Schelling, P. K.; Keblinski, P.; Cahill, D. G.; and Phillpot, S. R., "Phonon-defect scattering in doped silicon by molecular dynamics simulation" (2008). Faculty Bibliography 2000s. 1178. https://stars.library.ucf.edu/facultybib2000/1178

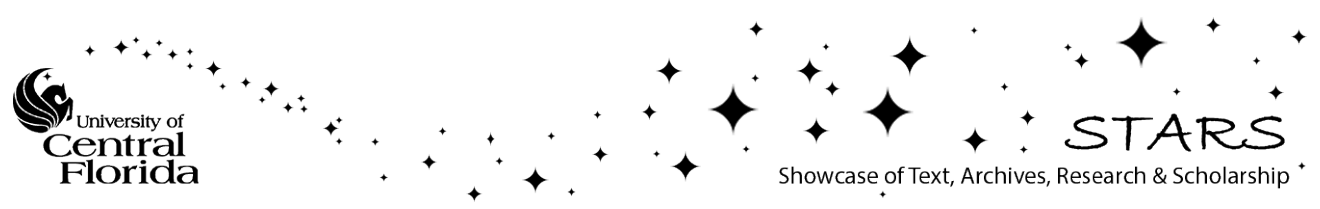


Authors

M. Yao, T. Watanabe, P. K. Schelling, P. Keblinski, D. G. Cahill, and S. R. Phillpot 


\section{Phonon-defect scattering in doped silicon by molecular dynamics simulation}

Cite as: J. Appl. Phys. 104, 024905 (2008); https://doi.org/10.1063/1.2959840

Submitted: 18 January 2008. Accepted: 24 May 2008. Published Online: 30 July 2008

M. Yao, T. Watanabe, P. K. Schelling, P. Keblinski, D. G. Cahill, and S. R. Phillpot

\section{ARTICLES YOU MAY BE INTERESTED IN}

Nanoscale thermal transport

Journal of Applied Physics 93, 793 (2003); https://doi.org/10.1063/1.1524305

Phonon wave-packet dynamics at semiconductor interfaces by molecular-dynamics simulation

Applied Physics Letters 80, 2484 (2002); https://doi.org/10.1063/1.1465106

Nanoscale thermal transport. II. 2003-2012

Applied Physics Reviews 1, 011305 (2014); https://doi.org/10.1063/1.4832615

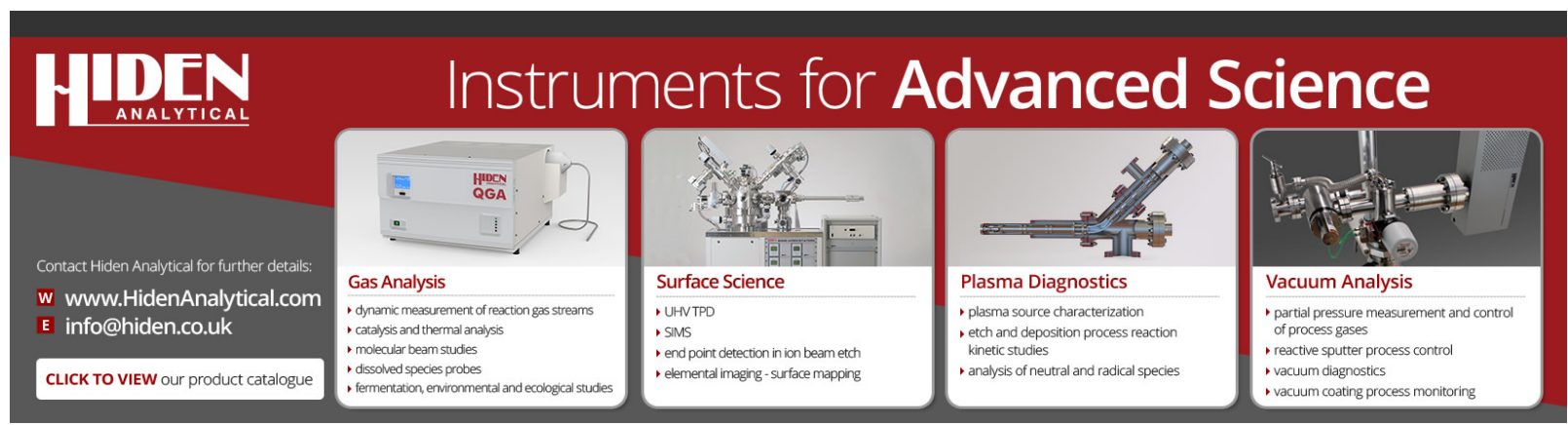




\title{
Phonon-defect scattering in doped silicon by molecular dynamics simulation
}

\author{
M. Yao, ${ }^{1,2}$ T. Watanabe, ${ }^{2}$ P. K. Schelling, ${ }^{3}$ P. Keblinski, ${ }^{4}$ D. G. Cahill, ${ }^{5}$ and S. R. Phillpot $^{2, a)}$ \\ ${ }^{1}$ School of Materials Science and Engineering, Dalian University of Technology, Liaoning 116024, \\ People's Republic of China \\ ${ }^{2}$ Department of Materials Science and Engineering, University of Florida, Gainesville, Florida 32611, USA \\ ${ }^{3}$ AMPAC and Department of Physics, University of Central Florida, Orlando, Florida 32816, USA \\ ${ }^{4}$ Department of Materials Science and Engineering, Rensselaer Polytechnic Institute, \\ Troy, New York 12180, USA \\ ${ }^{5}$ Department of Materials Science and Engineering, University of Illinois, Urbana, Illinois 61801, USA
}

(Received 18 January 2008; accepted 24 May 2008; published online 30 July 2008)

\begin{abstract}
Molecular dynamics simulations are used to study the scattering of phonon wave packets of well-defined frequency and polarization from individual point defects and from a field of point defects in Si. The relative amounts of energy in the transmitted and reflected phonon fields are calculated and the parameters that influence the phonon scattering process are determined. The results show that the fractions of transmitted and reflected energies strongly depend on the frequency of the incident phonons and on the mass and concentration of the defects. These results are compared with the classic formula for the scattering strength for point defects derived by Klemens, which we find to be valid when each phonon-defect scattering event is independent. The Klemens formula fails when coupled multiple scattering dominates. The phonon density of states is used to characterize the effects of point defects on mode mixing. (C) 2008 American Institute of Physics. [DOI: 10.1063/1.2959840]
\end{abstract}

\section{INTRODUCTION}

The scattering of phonons from point defects is one of the key mechanisms limiting the thermal conductivity of electrical insulators. These point defects range from isotopic defects to impurities to intentional dopants. The effect of point defects on the thermal transport properties is exemplified by diamond, in which isotopically purified diamond $\left(0.001 \%{ }^{13} \mathrm{C}\right)$ has a thermal conductivity at $80 \mathrm{~K}$ almost ten times higher than that of natural diamond $\left(1.1 \%{ }^{13} \mathrm{C}\right) .{ }^{1}$ This effect is also important in other materials. Particularly relevant for this study, the thermal conductivity of ${ }^{28} \mathrm{Si}$ epitaxial films is $16 \pm 5 \%$ larger than the thermal conductivity of natural $\mathrm{Si}^{2}$

Klemens ${ }^{3}$ analyzed the effects of a single isotopic defect and determined the inverse relaxation time for phonon scattering, $1 / \tau_{\text {single }}$, as

$$
\frac{1}{\tau_{\text {single }}}=\frac{a^{3}}{G}\left(\frac{m_{i}-m_{\mathrm{av}}}{m_{\mathrm{av}}}\right)^{2} \frac{\omega^{4}}{4 \pi v^{3}},
$$

where $\omega$ is the phonon angular frequency, $a^{3}$ is the atomic volume, $G$ is the number of atoms in the crystal, $\nu$ is the velocity of waves with $\nu=\omega / k$, and $k$ is the wave vector. In deriving Eq. (1), Klemens ${ }^{3}$ made the assumption that the material was a Debye solid. The $\omega^{2}$ in the Debye density of states $^{4}$ (DOS) leads to two powers of the frequency in Eq. (1), with the other two powers coming from the integration over $k$-vectors. The true densities of states of materials are significantly more complex than that of a Debye solid and

\footnotetext{
a) Author to whom correspondence should be addressed. Electronic mail: sphil@mse.ufl.edu.
}

can be expected to lead to a more complex phonon-defect scattering behavior.

More recently, Morelli et al. ${ }^{5}$ characterized the potential strength of isotopic defects in terms of the scattering coefficient $\Gamma$, which was defined in 1957 by Slack $^{6}$ as

$$
\Gamma=\sum_{i} c_{i}\left(\frac{m_{i}-m_{\mathrm{av}}}{m_{\mathrm{av}}}\right)^{2}, \quad m_{\mathrm{av}}=\sum_{i} c_{i} m_{i},
$$

where $c_{i}$ is the atomic fraction of a component $i$, whose mass $m_{i}$ deviates from the average, $m_{\mathrm{av}}$. Under this assumption that multiple dopants act as independent scatterers, the scattering rate depends linearly on the dopant concentration; Eq. (1) can then be rewritten for multiple scatters in terms of scattering coefficient $\Gamma$ as

$$
\frac{1}{\tau_{\text {multi }}}=\frac{a^{3} \Gamma}{4 \pi \nu^{3}} \omega^{4} \text {. }
$$

Cahill $\mathrm{et} \mathrm{al}^{7}$ recently measured the thermal conductivities of epitaxial layers of Si doped with up to 1 at. \% Ge and found that the dependence of the thermal conductivity on temperature and concentration is consistent with Eq. (3) even at these rather high dopant concentrations. This result is somewhat surprising because the Klemens ${ }^{3}$ formula is derived under the assumptions that the defects are isolated and isotopic in nature, neither of which is true for Ge-doped Si. Abeles ${ }^{8}$ demonstrated good agreement between experiment and theory using the Callaway model and scattering rate for point defects given by Eq. (3) in the high-temperature limit for alloys of $\mathrm{Si}_{x} \mathrm{Ge}_{1-x}$ with $x$ spanning the entire range from 0 to 1. This suggests that even for extremely high defect concentrations, the independent scattering model works at least reasonably well. 


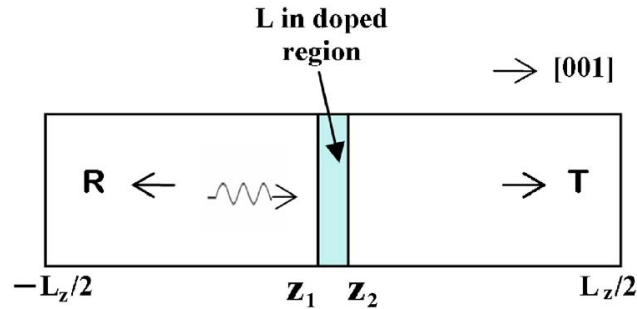

FIG. 1. (Color online) Schematic of the simulation cell showing the doped region. The fraction of energy reflected and transmitted are $R$ and $T$, respectively. The fraction of energy remaining in the dopant region is $L$.

In this paper, we use molecular dynamics (MD) simulation to explicitly simulate phonon-defect scattering processes. We determine the range of concentrations, relative masses, and frequencies over which the Klemens ${ }^{3}$ formula [Eq. (3)] is valid and identify the reasons for its breakdown.

The paper is organized in the following manner. Section II contains a brief discussion of the simulation methodologies. Phonon scattering from a single mass defect is discussed in Sec. III. Section IV elucidates the influence of concentration of dopant on phonon scattering. The frequency dependence for high dopant concentration is presented in Sec. V. The effects of mass difference, dopant field, and the size of the simulation cell on phonon scattering are addressed in Sec. VI; Sec. VII contains our final discussion.

\section{SIMULATION METHODOLOGY}

The simulation methods used here are similar to those that we have used previously for the simulation of phonon wave packet scattering at interfaces. ${ }^{9}$ The basic idea, shown in Fig. 1, is to construct a wave packet of phonons taken from a single branch and of a narrow frequency range. This wave packet is then launched from a region of perfect crystal at a point defect or into a field of point defects of welldefined concentration. This field of defects is taken to have a finite thickness, such that some fraction of the incident vibrational energy is transmitted, with the rest reflected. To monitor the scattering process itself, the time evolution of the energy transmitted through the dopant field is characterized, as are the energies reflected from it and the polarizations of both transmitted and reflected phonons. This wave packet dynamics is carried out using conventional MD methods. The incident phonons have sufficiently low amplitude that anharmonic coupling to other lattice modes is so weak that phonons propagate in the perfect crystal without any perceptible spreading or scattering; the ambient temperature of the system is $0 \mathrm{~K}$.

Silicon is used as a model system. For simplicity and to separate the isotope effect alone, the dopant atoms only differ from Si in having a mass four times larger. While such a mass difference is not of course physically achievable, it amplifies the isotope effect, making it more amenable to analysis. For real Ge-doped $\mathrm{Si}$ the ratio of atomic masses is 2.58 (i.e., $72.59 \mathrm{amu} / 28.09 \mathrm{amu}$ ). The interatomic interactions among the $\mathrm{Si}$ atoms and the dopant atoms are all described by the well-known and well-characterized Stillinger-Weber ${ }^{10}$ potential. ${ }^{11}$ The Si crystal is oriented such that the $x, y$, and $z$ axes correspond to the [100], [010], and

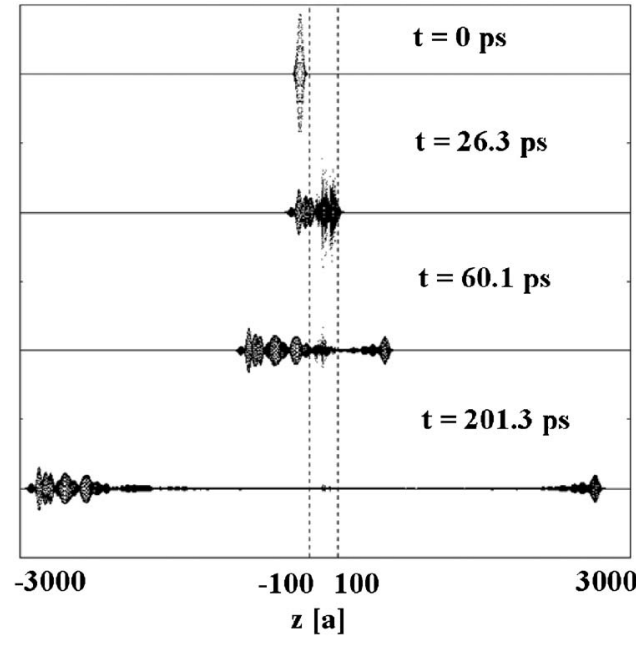

FIG. 2. Snapshots from the scattering of an incident LA phonon with frequency of $2.96 \mathrm{THz}$ from a region containing 1.56 at. \% concentration of dopants. The total length of the periodic simulation cell in the $z$ direction is $6000 a=3.26 \mu \mathrm{m}$; the doped region spans the region $-100 a<z<100 a$, i.e., has a thickness of $\Delta z=108.6 \mathrm{~nm}$.

[001] directions in the diamond lattice; the lattice constant is $a=0.543 \mathrm{~nm}$. The sides of the simulation cell have lengths of $L_{x}=L_{y}=2 a=1.08 \mathrm{~nm}$ and $L_{z}=1500 a$ and $6000 a$ (0.81 $\mu \mathrm{m}$ and $3.26 \mu \mathrm{m}$ ), depending on the specific simulation. Periodic boundary conditions are applied in all three spatial directions. Thus the system contains no surfaces; however as discussed below we take advantage of the small lateral dimensions to manipulate the phonon DOS and hence the phonon-defect scattering processes. As discussed at the appropriate points, for a few simulations we choose $L_{x}=L_{y}$ $=4 a$.

The doped region has a thickness $\Delta z=z_{1}-z_{2}$ (see Fig. 1). For $L_{x}=L_{y}=2 a$ the doped region of thickness $\Delta z=200 a$ (used in most of the simulations) contains a total of 6400 atoms; replacing $1,2,5,25,50$, and $100 \mathrm{Si}$ atoms with dopant atoms corresponds to concentrations of $0.016,0.031,0.08,0.39$, 0.78 , and 1.56 at. \%. The effect of the thickness of the doped layer is explored in Sec. VI.

The incident phonons are longitudinal acoustic (LA) phonons with frequencies $f$ ranging from 0.5 to $10 \mathrm{THz}$. Because the MD simulation places no restriction on the symmetry and frequency of the scattered phonons, the reflected and scattered contributions are analyzed so as to distinguish the LA from the transverse acoustic (TA) contributions. The energy of the incident LA phonons is not sufficient to produce optical phonons, so they need not be considered.

Figure 2 shows the amplitudes of the atomic displacements during a representative phonon scattering event, in this case for a dopant concentration of 1.56 at. \%. At $t=0$, the initial LA wave packet, with frequencies in the range of $0.025 \mathrm{THz}$ around a mean value of $2.964 \mathrm{THz}$ (i.e., 2.952$2.977 \mathrm{THz}$ ) and centered at $z=-250 a$, is launched toward the doped region. The leading edge of the wave packet reaches the doped region and hits the first dopant at $t=10.2 \mathrm{ps}$ (not shown). The leading edge of the unscattered component of the wave packet emerges from the dopant field at $t$ $=26.3 \mathrm{ps}$, by which time a reflected wave is already evident. 


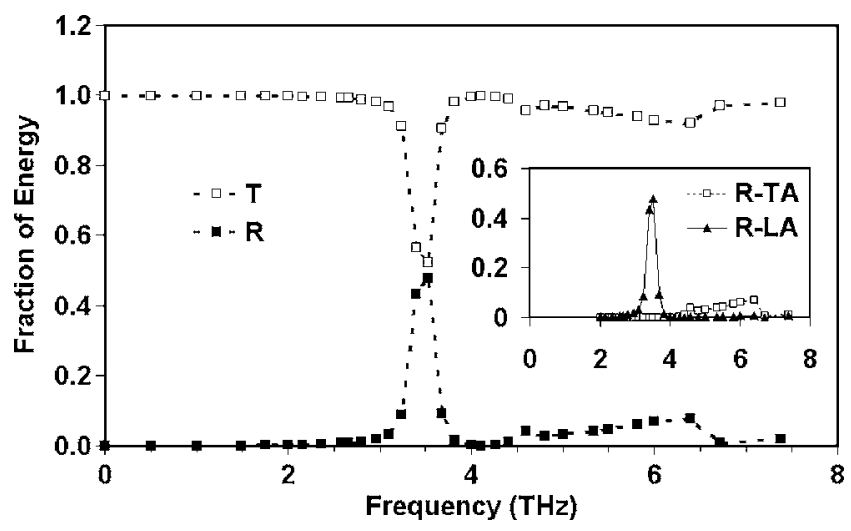

FIG. 3. Frequency dependence of transmission $(T)$ and reflection coefficients $(R)$ of phonon scattering from a single defect. The inset shows the fraction of the reflected energy in LA and TA modes. For all frequencies, the amount of trapped energy is negligible.

By $t=60.1$ ps a number of pulses of transmitted and reflected waves have emerged; however, there is still a significant amount of energy trapped in the doped region. By $t$ $=201.3 \mathrm{ps}$, the transmitted and reflected waves are approaching each other on the side of the periodic unit cell far from the doped region; at this time the simulation is stopped. Very little energy remains inside the defected region, allowing us to determine the reflection and transmission coefficients with high precision.

After this scattering process is over, the total energy $E$ of the incident phonon wave packet may be divided into three parts: the reflected energy $E_{r}$, the transmitted energy $E_{t}$, and the energy that remains trapped in the doped region $E_{L}$, as illustrated in Fig. 1. The fraction of reflected energy is defined as $R=E_{r} / E$, the fraction of transmitted energy as $T$ $=E_{t} / E$, and the fraction of trapped energy as $L=E_{L} / E$, with $R+L+T=1$.

The traditional theoretical analysis based on the Klemens ${ }^{3}$ result is posited in terms of the scattering time, whereas our results are naturally analyzed in terms of the reflection and transmission coefficients. While intuitively we expect them to be related, it is not a priori obvious that there is a linear relationship between them. However, as we shall see, the consistency of the analyses of our results will provide an a posteriori justification for treating the scattering time and the reflection coefficient essentially interchangeably.

\section{SCATTERING OF PHONONS FROM A SINGLE DEFECT}

As discussed in Sec. I, one of the predictions of the Klemens ${ }^{3}$ formula is that the phonon-defect scattering rate increases as the fourth power of the frequency. In this section we explicitly simulate the case of phonon scattering from a single dopant and analyze it in terms of the Klemens ${ }^{3}$ result.

Figure 3 shows the frequency dependence of $T$ and $R$ for a single defect. We see that they are complicated functions of the frequency. Generally, $T$ is close to unity for $f<3 \mathrm{THz}$ and for $f>4 \mathrm{THz}$ but drops to approximately 0.5 at around $3.5 \mathrm{THz}$.

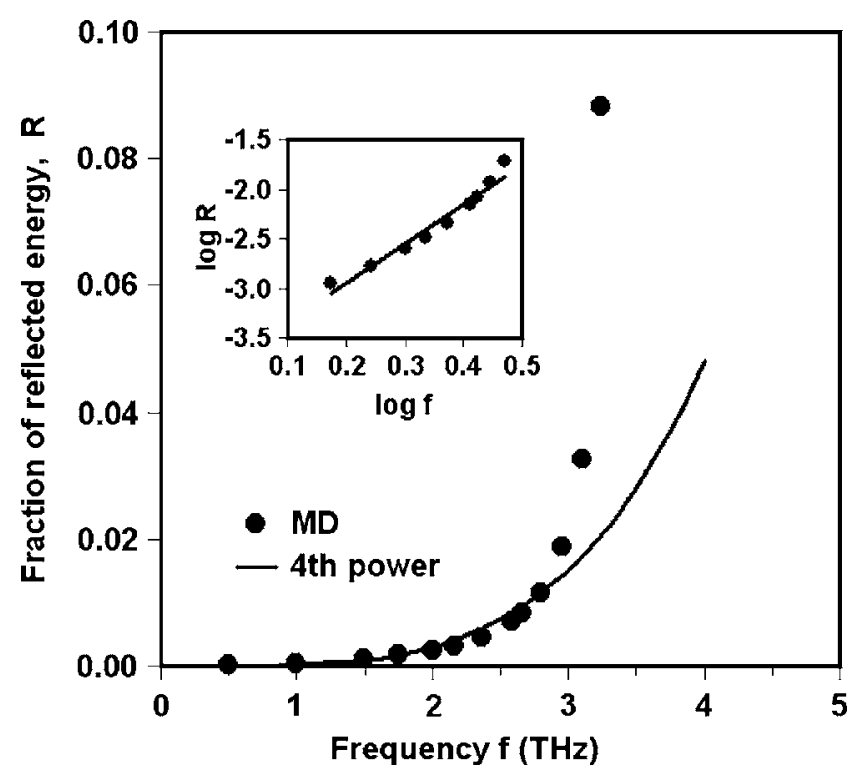

FIG. 4. Reflection coefficient at low frequencies and the best fit to a fourth power. The inset shows the log-log plot with a slope corresponding to a power exponent of 3.9 .

We first analyze the low-frequency regime $(f<3 \mathrm{THz})$. As Fig. 4 shows, in this regime, the reflection coefficient increases monotonically with frequency. Although the solid line clearly indicates that the frequency dependence of the reflection coefficient is close to the Klemens ${ }^{3} \omega^{4}$ dependence, the log-log plot in the inset, for which the best-fit exponent is 3.9 , shows that the data do deviate systematically from a simple power law. We thus conclude that in this lowfrequency regime, the Klemens ${ }^{3}$ result is reproduced at the semiquantitative level.

The most interesting region is $f=3-4 \mathrm{THz}$ (see Fig. 3), over which we see a considerable dip in the transmission coefficient, to a value of approximately 0.5 at $3.5 \mathrm{THz}$, with the reflection coefficient increasing to a similar value. To understand this reduction in the transmission coefficient, it is necessary to examine the phonon DOS in some detail. Figure 5(a) shows the calculated contribution from acoustic modes to the DOS of a large (i.e., essentially infinitely sized) single crystal. The DOS is determined by diagonalizing the dynamical matrix for a single unit cell for a set of wave vectors corresponding to the reciprocal lattice vectors of the supercell used in the dynamical simulations. $\mathrm{LA}_{\text {zero }}$ corresponds to LA modes in which $k_{x}=k_{y}=0$, of which there are so few that they cannot be seen on this figure. $\mathrm{LA}_{k x y}$ corresponds to LA modes for which $k_{x} \neq 0$ and/or $k_{y} \neq 0$. As expected, the DOS shows Debye-like behavior at lower frequencies; this DOS agrees well with that previously published for the Stillinger-Weber ${ }^{10}$ potential. $^{9,11}$

The size and shape of the simulation cell can have a significant effect on the DOS. In particular, the periodic system that we are using for our phonon/defect scattering simulations is $2 \times 2 \times N(N>2000)$. As shown in Fig. 5(b), the TA modes no longer show Debye behavior. Indeed, below 4 $\mathrm{THz}$, the only TA phonon modes present are the very few that correspond to wave vectors with $k_{x}=k_{y}=0$. However, at frequencies just above $4 \mathrm{THz}$, modes with nonzero $k_{x}$ and $k_{y}$ 

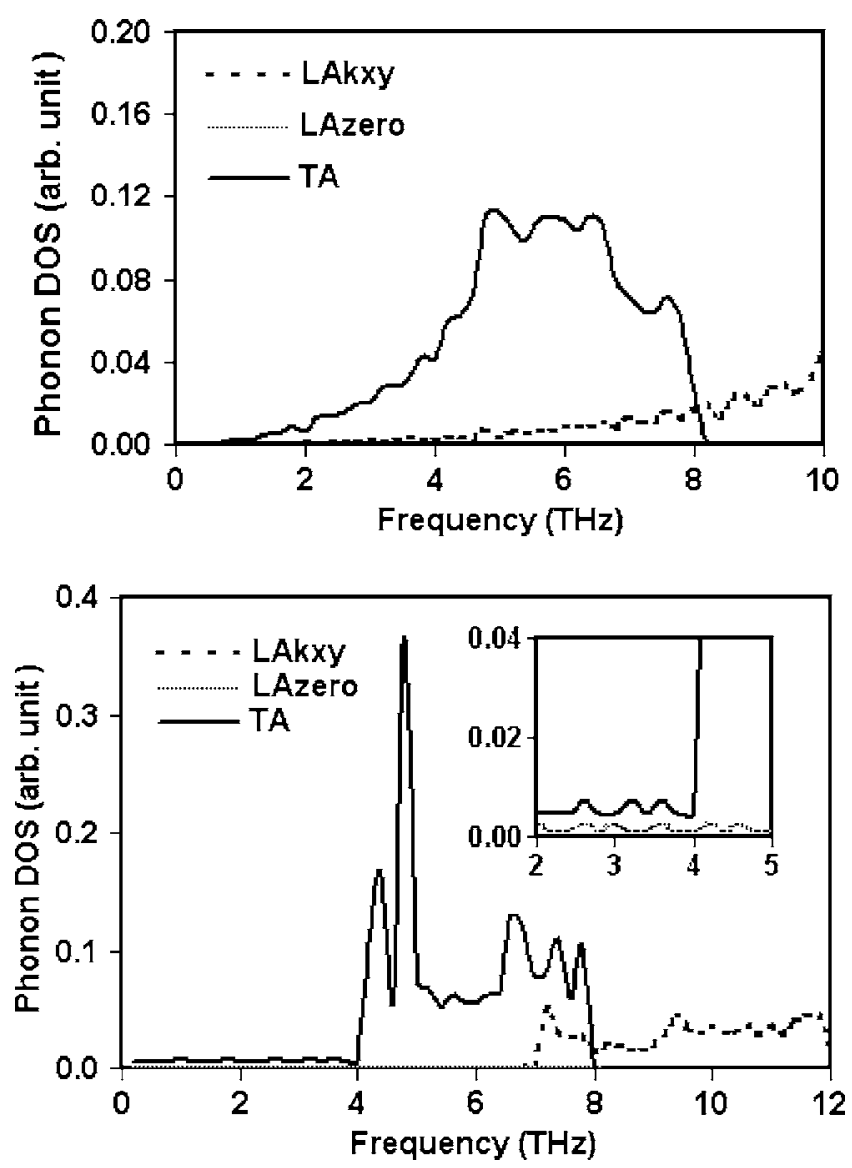

FIG. 5. Phonon DOS for (a) a large periodic perfect Si crystal (only shown up $10 \mathrm{THz}$ with LA and TA modes in which the DOS of $\mathrm{LA}_{\text {zero }}$ is too small to be visible) and (b) a perfect Si crystal with dimensions of $2 \times 2 \times N(N$ $=100)$.

begin to appear in the spectrum, resulting in a large increase in the DOS due to TA modes. In the absence of significant DOS in the region of $\sim 3.5 \mathrm{THz}$, the significant reduction in the transmission coefficient cannot thus be understood in terms of mode mixing between LA and TA modes.

The origin of the dip in the transmission coefficient actually lies with the dopants themselves. In particular, the dopants have a significant effect on the phonon DOS, as shown in Fig. 6, both for the $2 \times 2 \times N$ system [Fig. 6(a)] and for the large single crystal [Fig. 6(b)]. Of particular importance, in the frequency range of 3-4 THz, there are additional modes for the doped systems (green and red) that are not present in the perfect crystal (blue). To identify the origin of the modes, it is instructive to compute the local DOS (LDOS) associated with these modes. The LDOS at an atomic site $i$ for a given frequency $\omega$ is given by

$$
D_{i}(\omega)=\sum_{\lambda \mu} \varepsilon_{i \mu \lambda}^{*} \varepsilon_{i \mu \lambda} \delta\left(\omega-\omega_{\lambda}\right),
$$

where $\varepsilon_{i \mu \lambda}$ are the components of the phonon polarization vector with frequency $\omega_{\lambda}$ and $\mu$ labels the components of the phonon polarization vector. Figure 7, the LDOS for $f$ $=3.4-3.6 \mathrm{THz}$, indicates that these new modes are strongly associated with specific atoms in the system. For the small unit cell analyzed in this DOS analysis, $1.56 \%$ defect concentration corresponds to a single mass defect. Examination
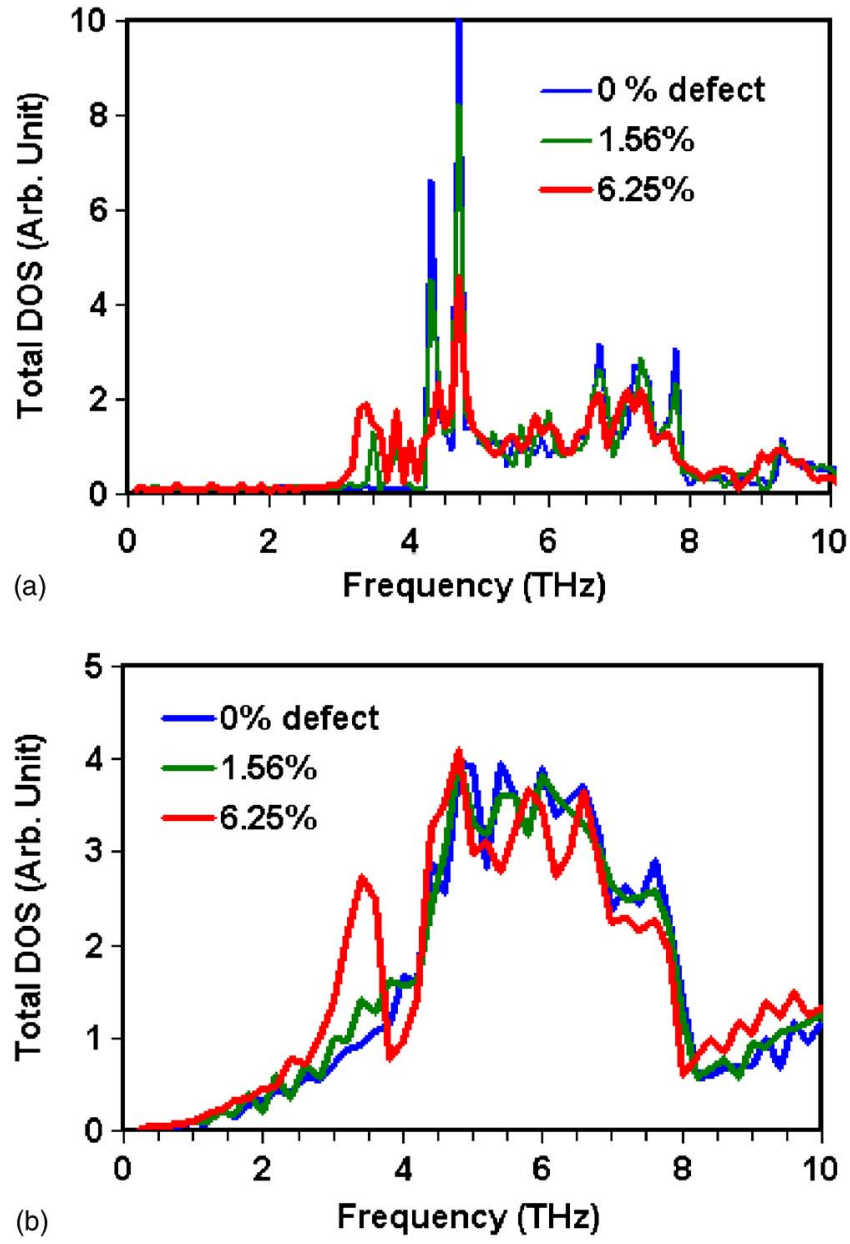

FIG. 6. (Color online) DOS as a function of dopant concentration for systems with sizes of (a) $2 \times 2 \times N,(N=100)$, and (b) $20 \times 20 \times 20$.

of the structure shows that atom 29 , at which there is a single strong peak, is the dopant atom, while the four smaller peaks (atoms 8, 25, 38, and 55) correspond to the first nearest neighbors of the dopant. There is thus a strong, spatially

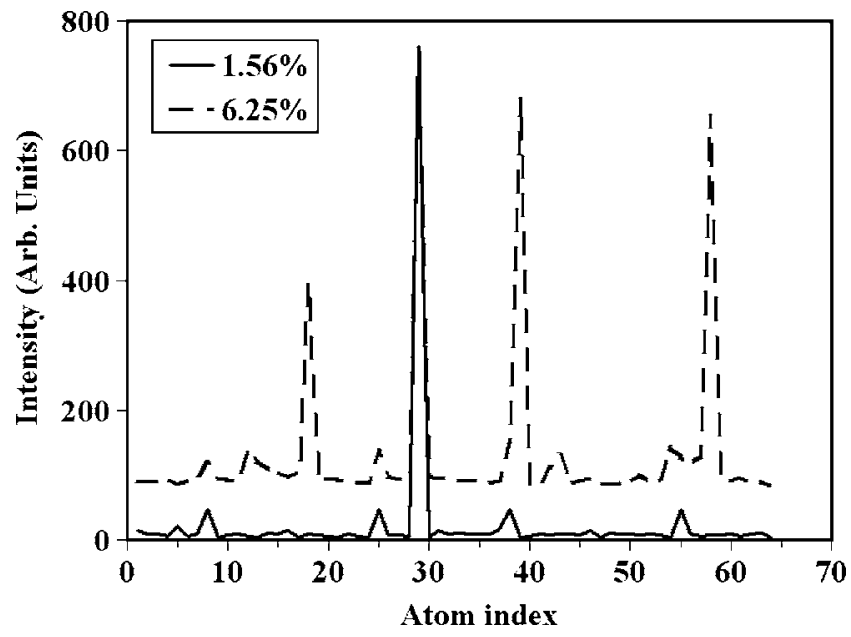

FIG. 7. Local DOS at $f=3.4-3.6 \mathrm{THz}$ associated with the dopant atoms. The locations of taller peaks correspond to the dopant atoms. For $1.56 \%$ doping (a single dopant out of 64 atoms) the four small peaks are contributed by four nearest neighbors of the dopant atom. For $6.25 \%$ the four tall peaks correspond to the dopant atoms. (For clarity the $6.25 \%$ curve has been shifted 80 units upward along the Intensity axis.) 
local resonant excitation in the region of the defect at this frequency. Such resonance scattering from a low-frequency local mode associated with the scattering of phonons by a localized perturbation in the lattice was previously seen by Klein. ${ }^{12}$ Moreover, Walker and Pohl ${ }^{13}$ observed that resonant scattering with the frequency lying in the acoustic continuum in a solid solution of $\mathrm{KCl}$ containing impurities resulted in a decrease in the thermal conductivity. We can then understand the strong change in the transmission and reflection coefficients as arising from the scattering of the LA mode from these point-defect resonant states. Also, as shown in Fig. 7, for $6.25 \%$ doping the four dopants randomly placed among $64 \mathrm{Si}$ atoms result in the four green peaks. The four large peaks, corresponding to the dopant atoms themselves, are of different heights because of their differing positions relative to each other. This large amplitude vibration in the proximity of the point defect will tend to couple directly to other phonon modes, with the results that the incident phonon is reemitted (i.e., scattered) in the backward and forward directions with almost equal amplitude.

At yet higher frequencies, the absence of these defect resonances might be expected to yield a return to Klemens-like ${ }^{3}$ behavior, with a transmission coefficient that continues to decrease strongly with frequency. However, the assumption of a Debye-like DOS is not valid in this frequency regime. Indeed, as Fig. 5(b) showed, for frequencies of 4-7 THz almost all of the DOSs arise from TA modes. We thus interpret the response on the system in this frequency regime as arising primary from scattering into the TA modes. This is evidenced in the inset to Fig. 3, which shows that essentially all of the reflected energies in the frequency range of $4-7 \mathrm{THz}$ is in the form of TA phonons. However, more than $85 \%$ of the transmitted energy is contributed as an unscattered LA mode; the remaining transmitted energy is in the form of TA vibrations. Since scattering into the TA modes involves a complete change in the nature of the vibrations, it is not surprising that very similar amounts of energy are contained in the transmitted and reflected TA channels.

Finally, the DOS in Fig. 5(b) shows that $\mathrm{LA}_{k x y}$ modes appear in the spectrum at $\sim 7 \mathrm{THz}$. These appear to offer an easier path to transmission than do the TA modes, as evidenced by the increase in the transmission coefficient to a value close to unity at this frequency (see Fig. 3).

In conclusion, this analysis shows that the complex frequency dependence of a single point defect on phonon propagation arises from a subtle interplay among the incident LA phonons, on the one hand, and the TA modes, $\mathrm{LA}_{k x y}$ modes, and defect resonance modes on the other. With this mechanistic understanding of the effects of phonon scattering from a single defect, we now examine the effects of multiple defects on the phonon-transport properties.

\section{DOPANT CONCENTRATION DEPENDENCE}

As a reference basis for the analysis of a system containing multiple dopants, we first estimate the transmission and reflection coefficients of a dopant field based on an independent scattering model. For randomly placed dopants, it is reasonable to assume that interference is incoherent, equally likely to be constructive and destructive, resulting in the scattering events being effectively independent of each other. However, as the dopant concentration increases, interference between successive scattering events can be expected to become more coherent, with the effect that the independent scattering model will break down. The sole inputs to this simple model are the above results for a single dopant. We also assume that transmission and reflection coefficients for each individual defect in a field of defects are the same as the single defect values, regardless of the actual concentrations of defects. The total transmission and reflection coefficients are then determined from the effects of multiple independent such scattering events. An essentially identical argument was previously used to predict the transmission coefficient for superlattices. ${ }^{14}$

Let $T_{1}$ and $R_{1}$ be the transmission and reflection coefficients of single defect, as calculated in Sec. III. For two scattering events, we obtain the formulas of $T_{2}$ and $R_{2}$ (with the subscript denoting the number of events) as ${ }^{14}$

$$
T_{2}=\frac{T_{1}}{1+R_{1}} \quad \text { or } \quad T_{2}=\frac{T_{1}}{2-T_{1}} .
$$

Likewise for $N$ scattering events,

$$
T_{N}=\frac{T_{1}}{N-(N-1) T_{1}} .
$$

Figure 8(a) compares the transmission coefficients calculated by Eq. (6) (solid lines) with the simulation results for frequencies of 2.96, 3.53, 3.68, and 5.5 THz. As shown in Fig. 8(a), the predictions of Eq. (6) are consistent with the results from multiple defects for low concentrations; for higher concentrations, the MD values depart significantly from the predictions.

We have analyzed the results in Fig. 8(a) in terms of the phonon wavelength and the mean distance between defects (easily determined from the concentration). With the bubble size indicating the deviation between MD results and independent scattering model, Fig. 8(b) shows a map in the space of phonon wavelength and mean defect separation in which three regions can be identified. The "independent scattering" regime corresponds to defect concentrations in which the predictions match the MD simulations to within 5\%. The intermediate scattering regime corresponds to the regime in which the independent scattering predictions are at least semiquantitatively correct (to within 5\%-50\%). The "strong scattering" regime then corresponds to that region in which the independent scattering fails significantly ( $>50 \%$ error). We see that the independent scattering model is well obeyed when the separation of defects is considerably larger than the phonon wavelength. As the defect separation decreases the scattering becomes stronger, resulting in larger differences between the results of $\mathrm{MD}$ and the independent scattering model when the separation of dopants is similar to or less than the phonon wavelength.

We now turn to direct simulations of the scattering from multiple dopants. Figure 9(a) shows $T$ and $R$ calculated from MD simulations as a function of concentration up to $1.6 \%$ for $f=2.96 \mathrm{THz}$. We can characterize this behavior in terms 

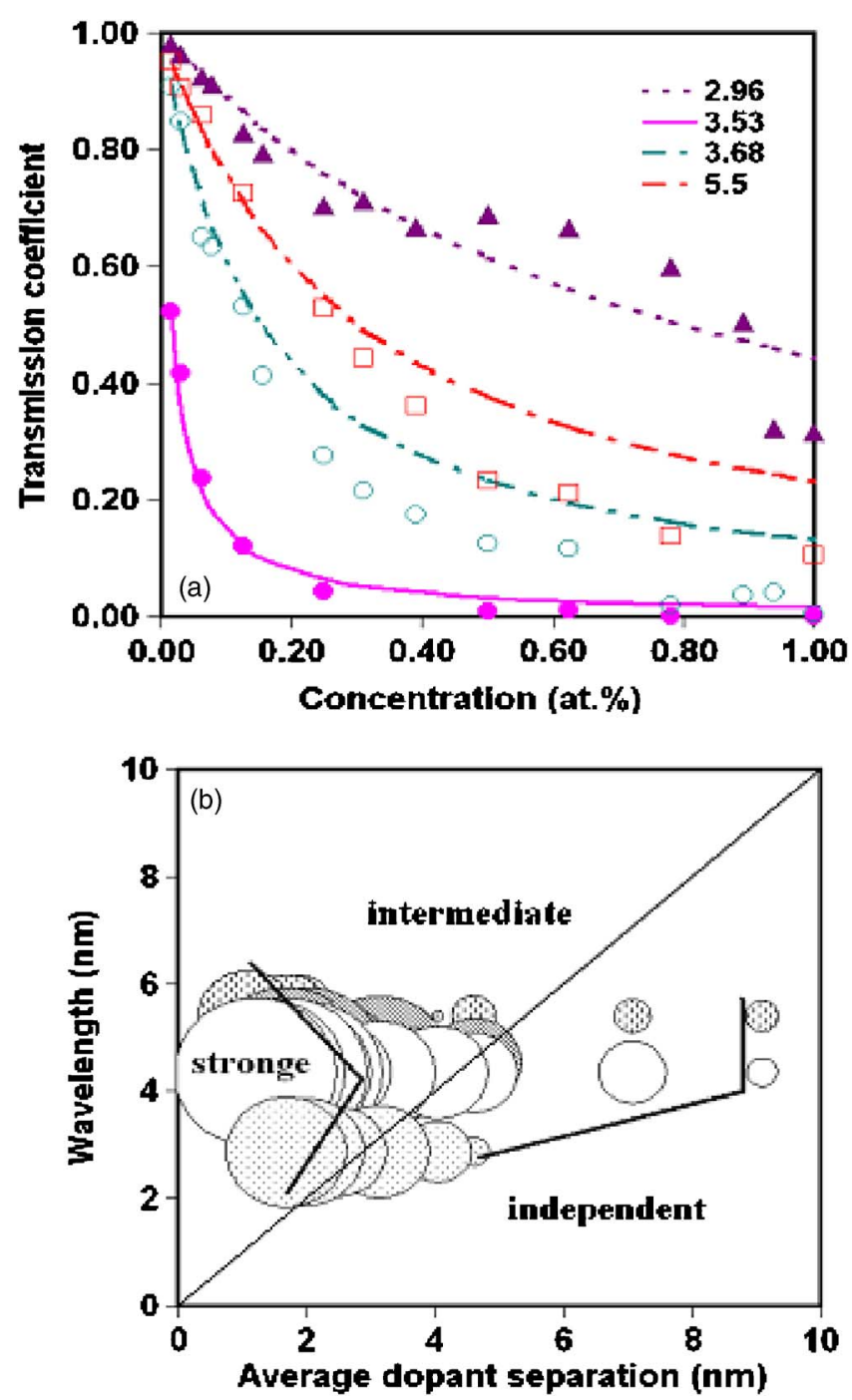

FIG. 8. (Color online) (a) Comparison of transmission coefficients directly from MD simulation (data points) and from the independent scattering model (lines) at various frequencies. The MD simulation results (data points) are for structures with 2, 4, 8, 16, 32, 50, and 64 dopants, corresponding to concentrations of $0.015,0.03,0.06,0.13,0.25,0.5,0.78$, and 1.0 at. \%, respectively. The legend is frequency in the unit of terahertz. (b) Bubble map in the space of phonon wavelength and average defect separation. The rightmost line shows the deviation of MD simulation and prediction by independent scattering model is less than $5 \%$. The leftmost line shows that the deviation is around 50\%. The diagonal shows the line for which the average defect separation is equal to the phonon wavelength.

of three regimes. The low-concentration region (up to $\sim 0.4 \%$ ) corresponds to the independent and intermediate scattering discussed above. In the second concentration regime, $0.4 \%-0.8 \%$, the transmission coefficient slowly decreases. Although not reproduced by the independent scattering model, this seems to be consistent with multiple scattering events. The third concentration regime, from $0.9 \%-1.6 \%$, is marked by the considerable drop in transmission coefficient at a concentration of $\sim 0.9 \%$, followed by a slow recovery with further increasing frequency. Figure 9(b) shows that this drop is also correlated with the onset of mode conversion from LA to TA modes in both reflection and transmission. The question is then why is this mode conversion not present at all concentrations? Analysis of the DOS again provides the answer. We saw in Fig. 6 that as the dopant concentration increases, the frequency of the local modes associated with the defects decreases. We thus interpret the onset of scattering into TA modes in Fig. 9(b) as arising from the lowest frequency defect state decreasing to $2.96 \mathrm{THz}$ at a concentration of $\sim 0.8 \%$. The nearly equal transmission and reflection coefficients from the TA modes in this regime are consistent with the phonons being scattered sufficiently often in the doped region that they lose any "memory" of where they came from. Thus, consistent with the diffuse mismatch model for phonon-interface scattering, the vibrational energy is emitted from the doped region according to the available DOS in the forward and backward directions. Because the doped region is surrounded by perfect crystal on both sides, the DOS is the same in both directions; thus the reflection and transmission coefficients for the TA modes should thus be equal, as they almost are. This mode mixing into the TA modes is accompanied by a significant drop in the LA transmission coefficient, as shown in Fig. 9(c). The increase in $T$ of TA modes and decrease in $T$ of the LA modes results in a nearly concentration independent transmission and reflection behavior in the high concentration regime $(c>\sim 1 \%)$.

To characterize the scattering process from a different perspective, we have determined the time over which the phonon wave packet actually interacts with the dopant field. The idea behind this is that the more scattering events the phonon wave packet undergoes, the more time it will remain in the dopant field; moreover, the more scattering events the wave packet undergoes, the more likely it is to undergo a significant amount of mode mixing. To precisely determine the scattering time, time is counted from the instant that the center of incident wave packet hits the first defect. Figure 10 shows the fraction of energy $L$ (the "trapped energy") remaining in the doped region at any given time as a function of the time. $L$ decreases as time increases, a result of the energy emerging from the dopant region into the transmitted and reflected packets. The data in Fig. 10 are reasonably well fit by the exponential function

$$
L=\exp \left(-\frac{t-t_{0}}{\tau}\right),
$$

from which we can define a trapping time $\tau$. Figure 11(a) shows the concentration dependence of $\tau$. We see that the trapping time tracks quite well with the reflection coefficient [line: taken from Fig. 9(a)], indicating that they are measuring essentially the same physical effect. This provides the promised a posteriori justification for the use of the reflection coefficient as a surrogate quantity for the scattering time.

The phonon diffusivity $D$ can be determined from the above scattering times obtained directly from the MD simulation,

$$
D=\frac{(\Delta z)^{2}}{\tau},
$$

where $\Delta z$ is the length of doped zone in the $z$ axis. An independent analytic estimate of the diffusion constant can be obtained from 

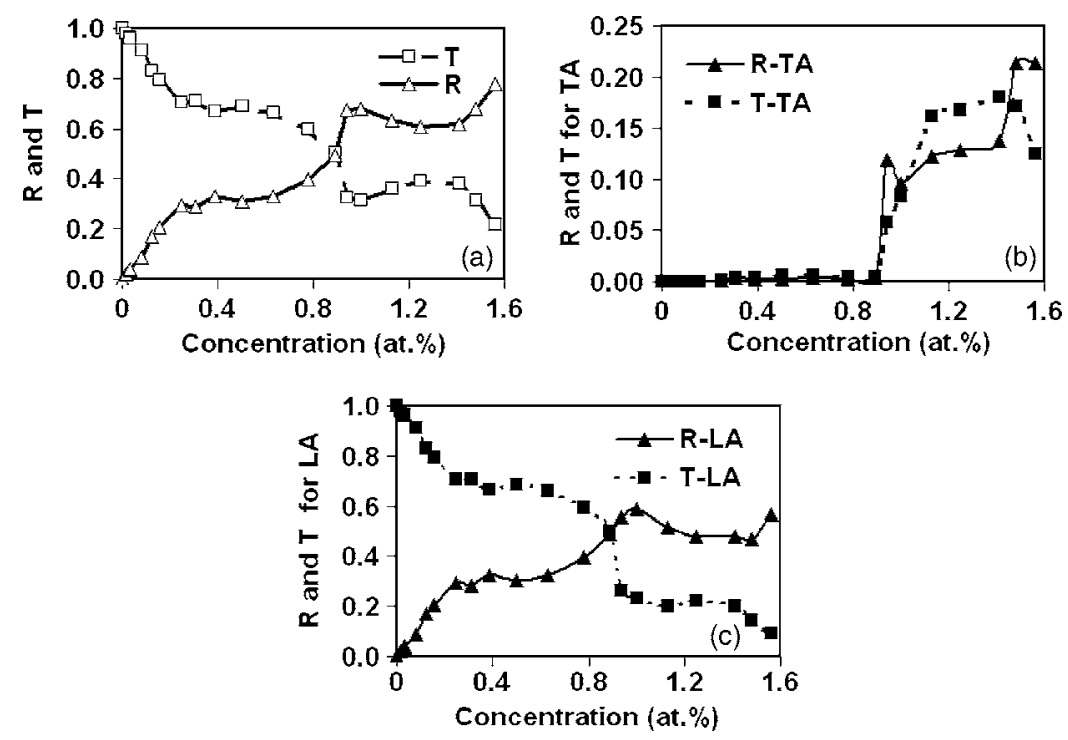

$$
D=\nu^{2} \tau_{\text {multi }}
$$

where $\nu$ is the group velocity of phonon, which is $\nu$ $=7896 \mathrm{~m} / \mathrm{s}$ for LA phonon of $2.96 \mathrm{THz}$. $\tau_{\text {multi }}$ can be calculated from Eq. (3). The two independent estimates of the diffusion constant are compared in Fig. 11(b). Given the rather simple analyses that yielded these estimates, the agreement between the two is very satisfactory: both are of a similar order of magnitude, and both show the same trend with concentration, albeit with the theoretical formula yielding consistently higher values.

\section{FREQUENCY DEPENDENCE FOR HIGH DOPANT CONCENTRATION}

We have seen that the Klemens ${ }^{3}$ formula gives the frequency dependence reasonably well for a single defect at low frequencies. In this section we examine the situation of high concentrations and high frequencies.

Figure 12(a) shows the frequency dependence of the transmission coefficient for defect concentrations of $0.25 \%$, $0.78 \%$, and $1.56 \%$ respectively. The response at lower frequencies is rather similar to that for the single dopant case. In particular, the transmission coefficient generally decreases at low frequencies. As shown in a log-log plot (Fig. 13) this

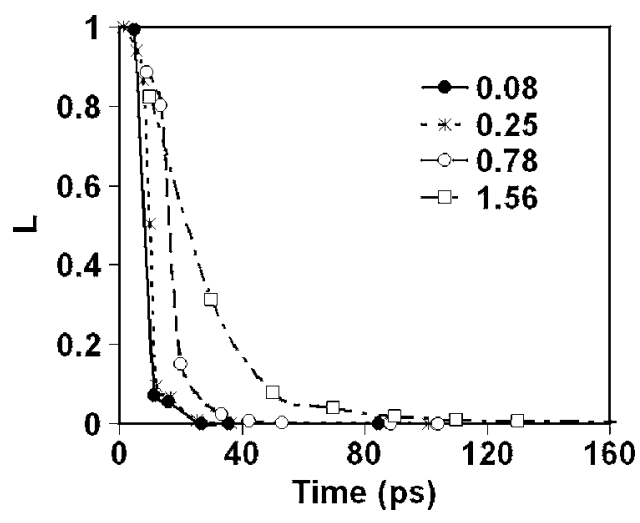

FIG. 10. Fraction $L$ of trapped energy as a function of the time. The labels are the dopant concentration in at. \% . The phonon frequency is fixed at 2.96 THz. low-frequency regime $(f<3 \mathrm{THz})$ is also reasonably well fitted by a power law. However, the power dependence is no longer close to the Klemens ${ }^{3}$ value of 4 , but 2.53 and 2.39 for $0.78 \%$ and $1.56 \%$, respectively. For comparison, the single defect case with the exponent of 3.9 is also reproduced from Fig. 4. These show that as the concentration increases, the frequency dependence of the phonon scattering decreases.

As in the single defect case, there is a significant drop in the transmission coefficient in the region of $3.5 \mathrm{THz}$. However, for these larger concentrations the effect is much larger, with the transmission coefficient actually dropping to almost zero, as compared to 0.5 for the case of the single dopant. These near-zero values for the transmission coefficient at 3.5 $\mathrm{THz}$ are a direct result of the 0.5 transmission coefficient for a single point defect that arises from the local resonant state.
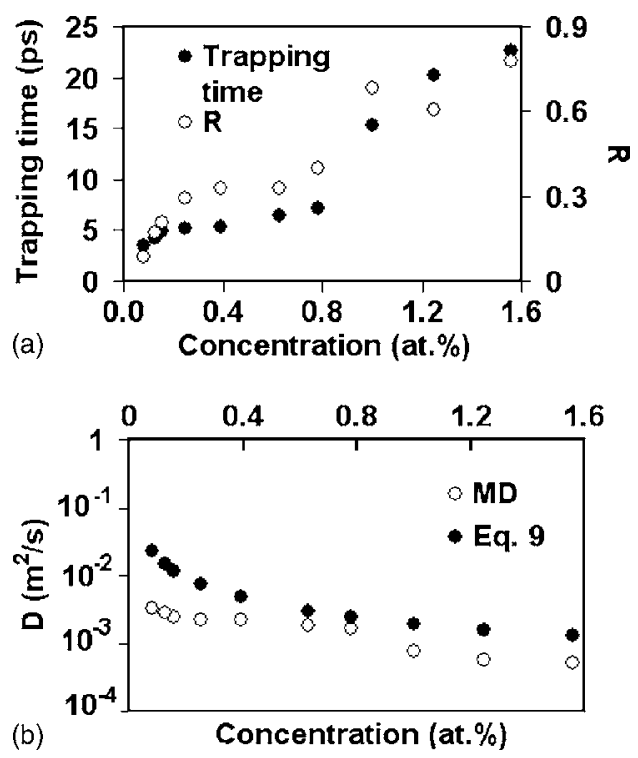

FIG. 11. (a) The phonon trapping time as a function of the concentration. The phonon frequency is fixed at $2.96 \mathrm{THz}$. For comparison the reflection coefficient $R$ taken from Fig. 9(a) as a function of the concentration is replotted. (b) The diffusion constants obtained by theoretical analysis via Eqs. (3) and (9) (solid symbols) and from the trapping time fit to the MD results via Eq. (8) (open symbols). 

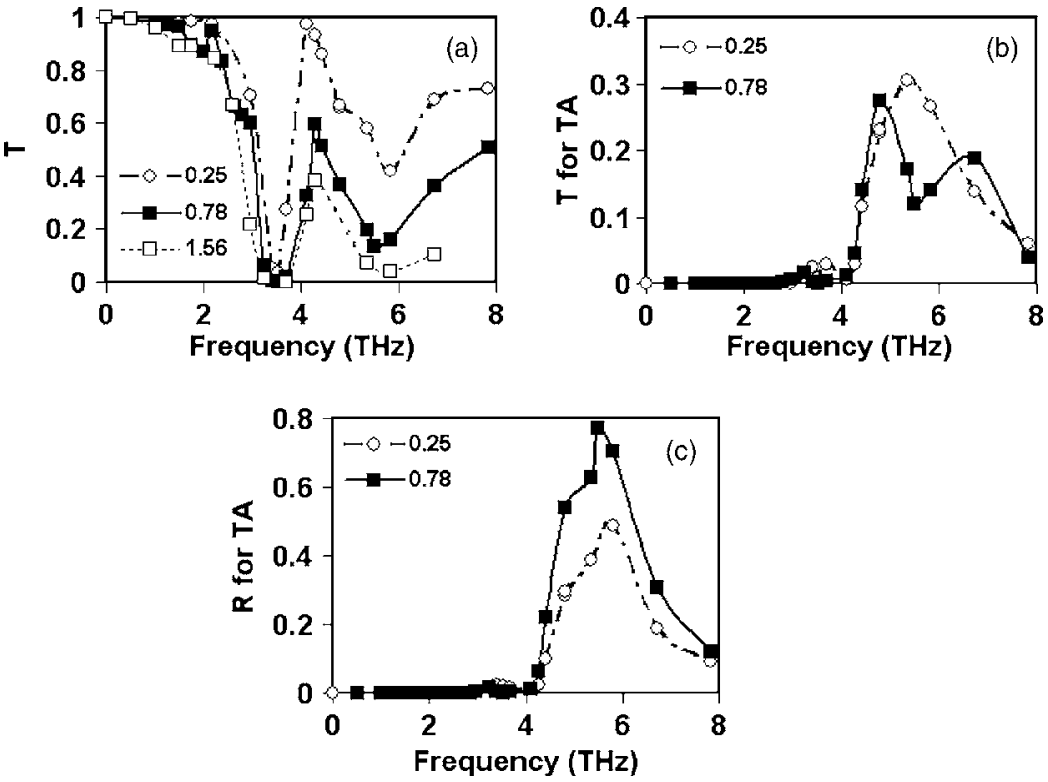

In particular $0.25 \%, 0.78 \%$, and $1.56 \%$ correspond to 16,50 , and 100 defects, respectively. For these concentrations, the independent scattering model of Eq. (6) yields transmission coefficients of $0.059,0.020$, and 0.01 . The actual transmission coefficients are actually somewhat lower, as we expect when the independent scattering approximation becomes poor: 0.038 for $0.25 \%$ and almost zero for $0.78 \%$ and $1.56 \%$. The increase in the transmission coefficient above the resonance is also analogous to that seen for the single dopant case and is again related to mode mixing. The new feature at these high dopant concentrations is the large drop in the frequency range above $4 \mathrm{THz}$. We can see in Figs. 12(b) and 12 (c) that the fraction of transmitted and reflected energies in the TA modes increases rapidly above $4 \mathrm{THz}$ (consistent with DOS in Fig. 5), reaching a maximum of $\sim 0.7$ at just below 6 THz. The increase in the transmission coefficient above $\sim 7 \mathrm{THz}$ is again largely the result of the availability of $\mathrm{LA}_{x y}$ modes in the DOS (see Fig. 5). It should be noted that $L$, the fraction of trapped energy, increases to more than $10 \%$ when

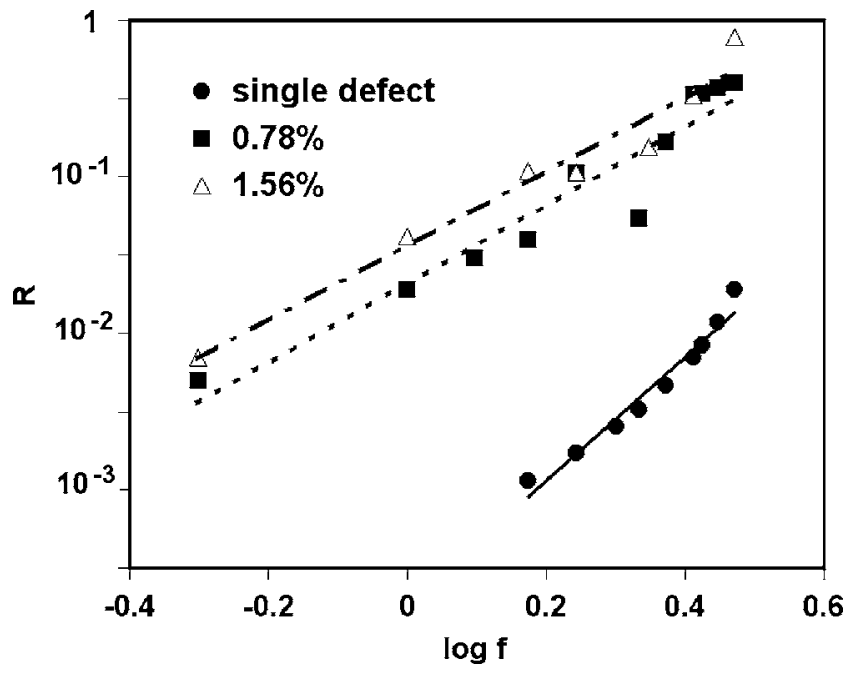

FIG. 13. Log-log plot of reflection coefficient vs frequency for $f<3 \mathrm{THz}$ and different concentrations. The single defect data are taken from the inset of Fig. 4. the concentration increases especially for 0.78 and 1.56 at. \%. Therefore, at higher frequencies (above $\sim 4 \mathrm{THz}$ ) the curve in Fig. 12 should be viewed more as a trend than as highly precise values for $T$ and $R$.

\section{EFFECTS OF MASS AND DISTRIBUTION OF DOPANTS AND SIMULATION CELL SIZE}

Up to this point, we have taken the mass of the dopant atoms to be four times that of Si. To explore the effect of different masses, we have also simulated systems with a dopant mass of only twice that of silicon. As is to be expected, the lighter dopants modify the phonon DOS to a lesser degree than the heavier dopants. In particular, as shown in Fig. 14(a), the lighter mass results in resonant states that are closer to the lower edge of the continuum states than for the heavier mass. The consequence of this is that the drop in the transmission coefficient due to the resonance is at a higher frequency, approximately $4 \mathrm{THz}$ as shown in Fig. 14(b), than it was for the heavier dopant. Moreover, this weaker dopant effect results in a somewhat smaller decrease in the overall transmission coefficient at the minimum.

For all of the studies described above, we arbitrarily chose the doped region to be of thickness 200a, and the distribution of dopants to be uniform within this range. In order to explore the effect of the thickness of the doped region, $\Delta z$, in Fig. 15 we compare the frequency dependence for two different thicknesses of dopant region, but for the same concentration. Reassuringly, the trends in $T$ are not affected by the thickness, albeit with the smaller $\Delta z$ giving a slightly larger $T$, which is reasonable since for a fixed concentration there are one-fifth the total number of dopants in the $40 a$ thick region than the $200 a$ region. We have also examined the effects of a graded rather than uniform distribution of dopants (not shown); we find that variations in the dopant distribution have little effect on the overall transmission and reflection coefficients.

All the results shown so far have been for a periodic simulation box of $L_{x}=L_{y}=2, L_{z}=6000(2 \times 2 \times 6000)$ unit 

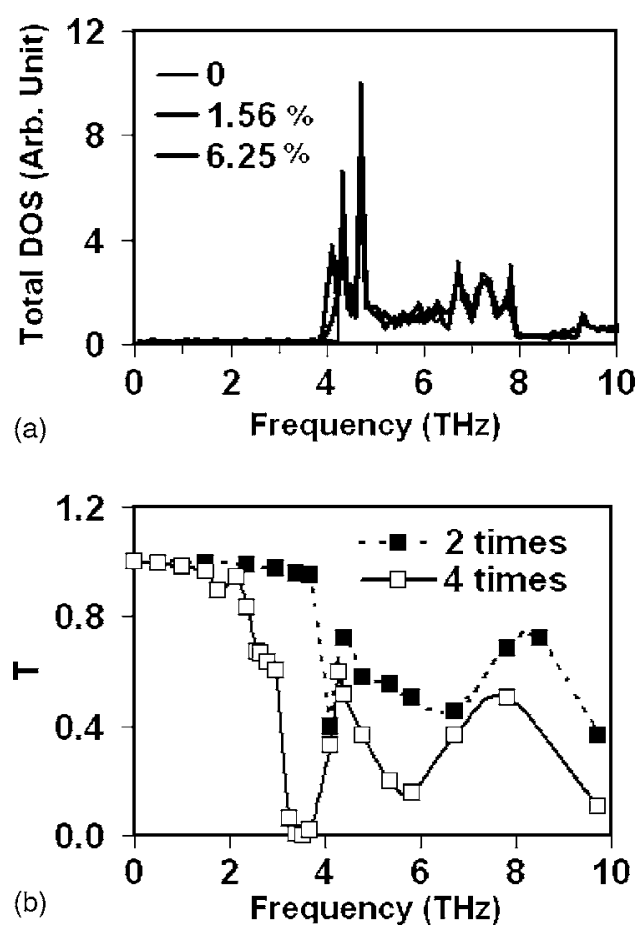

FIG. 14. Effect of atomic mass of dopants on the phonon scattering. (a) The DOS for dopant with two times the mass of Si with different dopant concentrations. (b) The fraction of total transmitted energy $T$ for $c$ $=0.78$ at. $\%$. The labels 2 times and 4 times correspond to the atomic mass for dopants that are two times and four times the atomic mass of $\mathrm{Si}$, respectively.

cells, in which the dopants are randomly distributed in a region of thickness $\Delta z=200 a$. It is also of interest to examine the effects of different choices of $L_{x}, L_{y}$ because, as Fig. 5 showed, these lateral dimensions have a significant effect on DOS. However, we need not be concerned with the effect of length in the $z$ direction since simulations show that increasing $L_{z}$ has no effect on the results.

To explore the effect of the area of a simulation cell, we use a simulation cell of $4 \times 4 \times N$ instead of $2 \times 2 \times N$. Figure 16(a) shows the DOS for the $4 \times 4 \times N$ system; here there

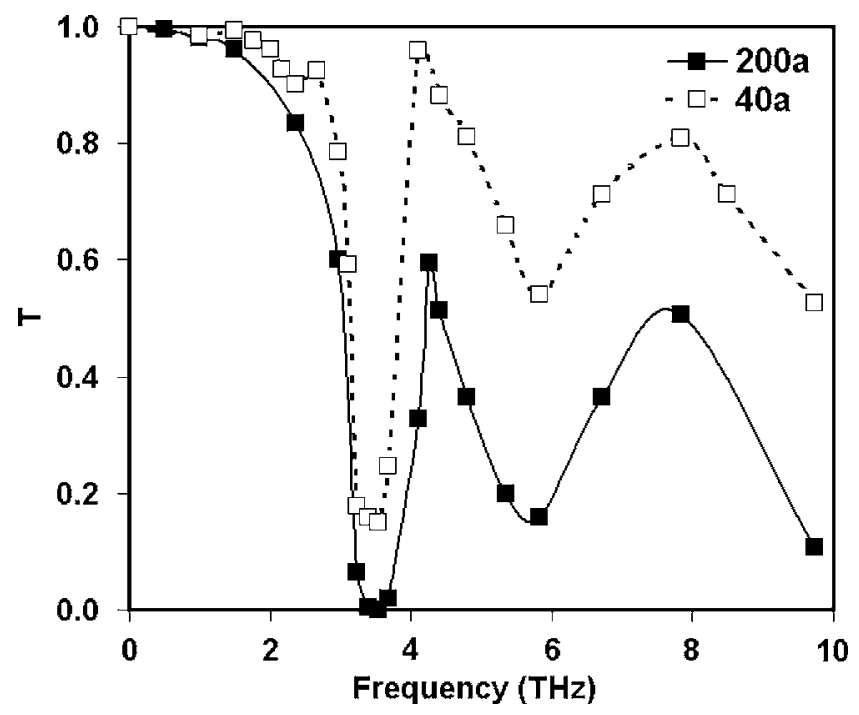

FIG. 15. The effect of the thickness of the dopant region $\Delta z(0.78$ at. \%) on the fraction of total transmitted energy $T$.
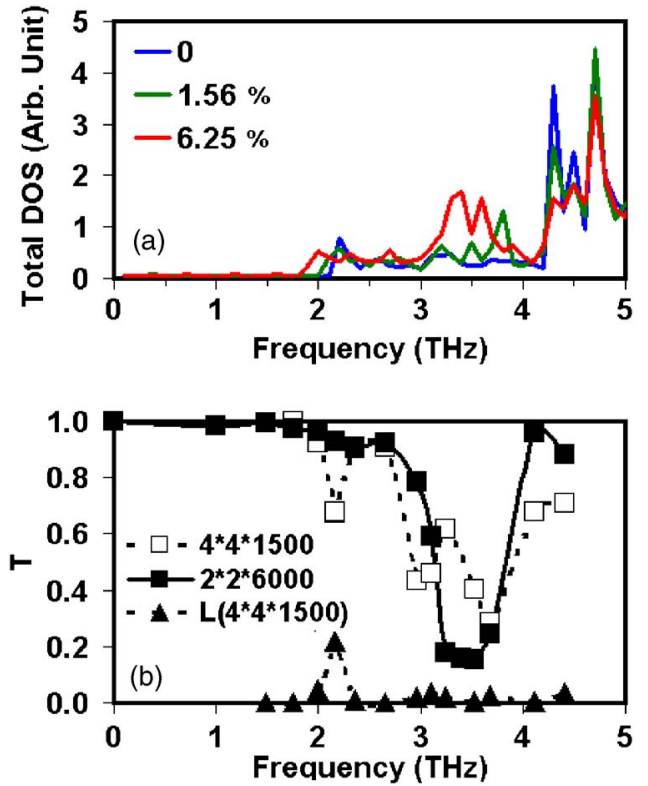

FIG. 16. (Color online) The effect of the area of the simulation box size in the $x$ and $y$ directions. (a) The DOS for the $4 \times 4 \times 100$ system with and without defects (show only up to $5 \mathrm{THz}$ ). (b) The transmission coefficients for the $2 \times 2 \times 1500$ and $4 \times 4 \times 1500$ systems $(c=0.78$ at. $\%, \Delta z=40 a) . L$ denotes the fraction of trapped energy.

are new states at frequencies below those previously present in the $2 \times 2 \times N$ DOS. However, the dopants still result in additional contributions to the DOS in the frequencies around $3.5 \mathrm{THz}$, plus an additional contribution below the lowest continuum state at $\sim 2 \mathrm{THz}$. This modifies the scattering behavior of the systems, as shown in Fig. 16(b). In particular, there is now an additional decrease in the transmission coefficient just above $2 \mathrm{THz}$, which is consistent with the presence of phonon states in this frequency range that were absent in the $2 \times 2 \times N$ system. To understand the results in Fig. 16(b), the local DOS with $6.25 \%$ dopant concentration for the $4 \times 4 \times 100$ system were determined at 2.0-2.2, 2.9-3.1, and 3.6-3.8 THz, which correspond to the three dips in the transmission coefficient in Fig. 16(b) for the $4 \times 4 \times 100$ system. In each case the LDOS showed peaks on the dopant atoms and their neighbors, confirming that the dips are resonant effects.

\section{DISCUSSION}

This study provides a detailed analysis of the domain of applicability of the Klemens ${ }^{3}$ analysis of phonon-defect scattering. The Klemens ${ }^{3}$ analysis is predicated on the independent scattering of phonons from isolated defects in the absence of mode mixing. By constraining the cross section of the simulation cell, by looking at low frequencies, and by exploring the low-concentration limit, we constructed the simulations to come as close to these conditions as possible. For this somewhat artificial scenario we have indeed established that Klemens, 3 prediction of $\omega^{4}$ dependence of the scattering rate is obeyed, at least semiquantitatively. However, more realistic simulation conditions lead to both quantitative and qualitative breakdowns in this behavior. First, we found that scattering events are only independent of each 
other if the separation of the defects is at least of the order of the phonon wavelength, a situation that is reasonably well obeyed for low defect concentrations. This, however, leads to a decrease in the exponent of the Klemens ${ }^{3}$ frequency dependence but does not lead to the complete breakdown of Klemens-like ${ }^{3}$ behavior. Second, mode mixing at low frequencies was suppressed by using a simulation cell with a very small cross section, thereby cutting off the lowfrequency TA modes. However, we saw that for simulation cells with larger cross sections, the frequency range in which Klemens-like ${ }^{3}$ behavior is seen was significantly reduced. Third, the introduction of resonant modes in the vibrational properties due to the presence of the dopant is not accounted for in the Klemens ${ }^{3}$ model. As we have also seen, these resonant modes can lead to a significant modification of the phonon-defect scattering behavior over a frequency range of $\sim 1 \mathrm{THz}$ or more.

Finally, returning to the experiments that motivated this work, the atomic mass ratio of dopant $\mathrm{Ge}$ to $\mathrm{Si}$ is 2.58 and the highest dopant concentration studied was 1.0 at. \%. Following the idea that Klemens-like ${ }^{3}$ independent and intermediate scattering regimes are valid for lower dopant concentration and for frequencies below the resonant frequency, they can be expected to over a wider frequency and concentration domain for Ge-doped Si than for our model defects. We may thus interpret the experimental behavior of the Gedoped Si as predominantly arising from phonon-defect interactions that correspond to the independent and intermediate scattering regimes.

\section{ACKNOWLEDGMENTS}

The work of M.Y. was supported by the Chinese Scholarship Council. The work of T.W. was supported by DOENERI Award No. DE-FC07-05ID14649. The work of P.K.S. was supported by NASA-Glenn National Laboratory and Sandia National Laboratory. The work of D.G.C. was supported by ONR Grant No. N00014-05-1-0250. The work of S.R.P. was supported by DOE-NERI Award No. DE-FC0705ID14649 and by the American Chemical Society Petroleum Research Fund. We thank the High Performance Computing (HPC), University of Florida, for providing resources for our calculations.

${ }^{1}$ L. H. Wei, P. K. Kuo, R. L. Thomas, T. R. Anthony, and W. F. Banholzer, Phys. Rev. Lett. 70, 3764 (1993).

${ }^{2}$ D. G. Cahill and F. Watanabe, Phys. Rev. B 70, 235322 (2004).

${ }^{3}$ P. G. Klemens, Proc. Phys. Soc., London, Sect. A 68, 1113 (1955)

${ }^{4}$ C. Kittel, Introduction to Solid State Physics, 8th ed. (Wiley, Hoboken, NJ, 2005).

${ }^{5}$ D. T. Morelli, J. P. Heremans, and G. A. Slack, Phys. Rev. B 66, 195304 (2002).

${ }^{6}$ G. A. Slack, Phys. Rev. 105, 829 (1957).

${ }^{7}$ D. G. Cahill, F. Watanabe, A. Rockett, and C. B. Vining, Phys. Rev. B 71, 235202 (2005).

${ }^{8}$ B. Abeles, Phys. Rev. 131, 1906 (1963).

${ }^{9}$ P. K. Schelling, S. R. Phillpot, and P. Keblinski, Appl. Phys. Lett. 80, 2484 (2002).

${ }^{10}$ F. H. Stillinger and T. A. Weber, Phys. Rev. B 31, 5262 (1985).

${ }^{11}$ J. Q. Broughton and X. P. Li, Phys. Rev. B 35, 9120 (1987).

${ }^{12}$ M. V. Klein, Phys. Rev. 131, 1500 (1963).

${ }^{13}$ C. T. Walker and R. O. Pohl, Phys. Rev. 131, 1433 (1963).

${ }^{14}$ P. K. Schelling and S. R. Phillpot, J. Appl. Phys. 93, 5377 (2003). 